response to treatment with phorbol ester. It was therefore natural to conjecture that the cellular homologue of JUN might be AP-1.

Bohmann et al. in their new work ${ }^{2}$ show that this is indeed the case. Antibodies to JUN crossreact with AP-1. The cellular homologue of the jun gene, c-jun, has been isolated from a human genomic library and shown to encode six peptides which form part of AP-1. Finally, a complementary DNA version of c-jun, when expressed in bacteria, produces a protein with DNA-binding properties virtually indistinguishable from those of AP-1. The conclusion that c-jun really is the gene encoding AP-1 seems unavoidable. On the other hand, the differences between jun and c-jun are considerable, and suggest that JUN is not impersonating a simple AP-1 so much as an AP-1 that has bound phorbol esters. If so, this would confirm the earlier idea $a^{1}$ that the cell hosting JUN might be permanently subject to some of the effects of these tumour promoters. A forthcoming report from Michael Karin's group ${ }^{3}$ (which also confirms much of the work of Bohmann et al.) supports this idea by showing that JUN activates transcription strongly in transfected cells without the need for phorbol esters.

Although this is significant in itself, some of the avenues of enquiry it suggests could be even more interesting. One of these is whether there may not be other proteins that bind to the AP-1 site. Bohmann et al. ${ }^{2}$ mention that they have found at least one other gene with homology to jun, and that another group has found a third such gene in mice. Moreover, when AP-1 is purified on affinity columns bearing its binding site, the result is a preparation containing one major and three minor polypeptides. It therefore seems probable that JUN belongs to a multigene family, all the members of which have very similar DNA-binding domains and recognize the same sequence. The common DNA-binding domain plainly has a very long evolutionary history in this case, as it can still replace its yeast counterpart in GCN4. Despite this, the remaining parts of JUN and GCN4 bear no resemblance to each other, and there is no counterpart in JUN for the acidic amino acids that activate yeast transcription ${ }^{4}$. This evolutionary conservatism thus appears to be a case of Walter Gilbert's "exon shuffling", resembling the relationship between $c d c 10$ in fission yeast and Notch in fruitflies (which share a domain but not, so far as is known, any function ${ }^{6}$ ) rather than the relationship between the $c d c 2 \mathrm{~s}$ in fission yeast and humans (which share both structure and function ${ }^{7}$ ). DNA-binding domains seem to have a particular predelection for being shuffled; this characteristic has been used, for instance, to isolate several genes from mice, all pos-

\title{
SN1987A pulsar slow to reveal itself
}

THE light curve of supernova 1987A has been falling now for several months, following an exponential law, with a half-life of 78 days. This is convincing evidence that the power behind the luminosity is the radioactive decay of ${ }^{56} \mathrm{Co}$ to ${ }^{56} \mathrm{Fe}$; the ${ }^{56} \mathrm{Co}$ comes from the rapid decay of ${ }^{56} \mathrm{Ni}$, about half a solar mass of which was synthesized in the original explosion. At the recent meeting of the American Astronomical Society in Austin, Texas (10-15 January 1988), direct evidence for the radioactive decay model was presented in the detection by G. Share et al. (reported in full on page 416 of this issue) of characteristic $\gamma$-ray lines of ${ }^{56} \mathrm{Co}$. Moreover, the light curve is beginning to show signs of a slight turndown below the exponential curve, as expected: when $\gamma$-rays from the decay start to leak through the thinning shell, less energy is available to be degraded into optical emission.

Nucleosynthesists in Austin were evidently pleased that $\mathrm{SN1987A}$ is following their calculations with such admirable precision. But there are a few puzzles. The brightness in some parts of the ultraviolet is increasing, and IAU circulars 4530 and 4532 report increases in X-ray emission in the 6-28 keV energy range.

Advocates of the pure-radioactivity model attribute the ultraviolet emission to fluorescence of a shell of circumstellar material. This shell would be the result of mass loss by the supernova progenitor in an earlier red-giant phase, and radiation from the explosion will only just reach it to excite the fluorescence.

The same advocates generally ascribe the observed soft X-rays to experimental error; more particularly to contamination of the observations by the powerful source LMC X-1, not far in the sky from SN1987A. But another explanation is that the various increases in emission signal the emergence of a second power source - a pulsar.

Everyone agrees that a neutron star is lurking within the supernova remnant, and if it is spinning and has a magnetic field it

sessing the so-called 'zinc finger' DNAbinding domain and so presumably involved in transcriptional regulation ${ }^{8}$. Nevertheless, no proteins other than the AP-1s are yet known to share the GCN4binding domain, and it remains to be seen whether this element can be modified so as to recognize different DNA sequences in different proteins.

Whether or not it can, the idea that there is a family of AP-1s makes the transcriptional response to phorbol esters and its relationship to carcinogenesis seem even more complicated than it did already. The effect of phorbol esters on the other AP-1s is not yet known, but at least four further transcription factors have been described whose effectiveness must transfer energy to the nebula. The mechanism and efficiency of this transfer are hard to guess at this early stage of development, but as radioactive decay declines and is replaced by a constant energy supply from the pulsar, the light curve should eventually level out.

There is no sign yet of this happening, and the light level is now comparable to the output of the Crab Nebula, which is entirely powered by its central pulsar. If the decline continues, pulsar theorists will begin to worry, although the birth of pulsars is a complex and poorly understood process, so that escape clauses can be found. It may be, for example, that pulsars are not born with a strong magnetic field but generate one by an internal dynamo mechanism, so that the pulsar will gradually turn on as the months pass.

Observers are also looking out for more specific signs of the presence of a pulsar. Good evidence, according to Alice Harding of NASA/Greenbelt, would be the detection of ultra-high-energy $\gamma$-rays, perhaps up to $10^{15} \mathrm{eV}$, from the supernova remnant. The spinning magnetic field of the pulsar is able to accelerate charged particles, mostly electrons and protons, to these enormous energies; the protons collide with atoms in the supernova ejecta, creating, among other things, neutral pions which decay rapidly into $\gamma$-ray photons, some of which are able to escape the nebula and reach us. This is a lengthy chain of events, but it is widely accepted that only a pulsar is capable of generating such huge energies in individual particles, and their detection by atmospheric air showers or Cerenkov radiation on Earth would be proof for most astronomers that a pulsar exists.

Concern over the absence of a direct sighting of the pulsar has not yet turned to embarrassment, but the next few weeks and months, as energy from radioactivity falls off, will be crucial. A prolonged silence may cause even the most stalwart friends of pulsars to think about revising their ideas. David Lindley

is increased by these compounds 9 . Are any of these in fact the products of multigene families? How do the factors in this vast array interact with one another? And which, if any, of them are potential oncogenes like c-jun? There are plainly many interesting answers to look forward to.

1. Short, N.J. Nature 330, 209-210 (1987).

Bohmann, D. et al. Science 238, 1386-1392 (1987)

Angel, P. et al. Nature (in the press).

Ma. J. \& Ptashne, M. Cell 51, 113-119(1987)

Gilbert, W. Science 228, 823-824 (1985).

Breeden, L. \& Nasmyth, K. Nature 329, 651-654 (1987)

Lee, M.G. \& Nurse, P. Nature 327, 31-35 (1987).

Chowdhury, K. et al. Cell 48, 771-778 (1987).

9 Chiu, R et al. Nature 329, 648-651 (1987).

N.J. Short is in the Department of Biophysics, Cell and Molecular Biology, King's College London (KQC), 26-29 Drury Lane, London WC2B SRL, UK. 